\title{
A Blind Print-Recapture Robust Watermark Scheme by Calculating Self-Convolution
}

\author{
Mengmeng Zhang, Beijing Key Laboratory of Advanced Information Science and Network Technology, Beijing Jiaotong \\ Univ, Beijing, China \\ Rongrong Ni, Beijing Key Laboratory of Advanced Information Science and Network Technology, Beijing Jiaotong Univ, \\ Beijing, China \\ Yao Zhao, Beijing Key Laboratory of Advanced Information Science and Network Technology, Beijing Jiaotong Univ, \\ Beijing, China
}

\begin{abstract}
A blind print-recapture robust watermark scheme is proposed. Watermark patterns are embedded into the space domain of a color image and can be detected from a print-recaptured version of the image without knowledge of the original image. The process of embedding invisible watermarks to convert RGB color images to CIE Lab color spaces and embed periodic watermarks in both color channels at the same time. Watermark extraction is achieved by calculating self-convolution and inverting the geometric transformation such as rotation and scale. Normalized correlation coefficients between the extracted and the embedded watermark pattern is calculated to determine whether there is watermark. The decision about the presence/absence of the watermark pattern is then determined by a threshold which is set 0.13 , and the detection rate of 241 pictures is about 0.79 .
\end{abstract}

\section{KEYWORDS}

Blind Detection, Digital Watermark, Geometric Distortion, Print-Recapture Watermark, Robust Watermark

\section{INTRODUCTION}

Digital watermark technology is to embed a small amount of information (called watermark) into digital signal without affecting its original value of use. The embedded information is usually imperceptible to the human eye, and can be detected or extracted by specific calculation. The watermark is combined closely with the original signal and hidden there, becoming an inseparable part of it. Digital watermarking technology is one of the effective means for digital media copyright protection and content integrity verification.

The rapid development and wide application of network technology have facilitated the acquisition, storage, distribution and use of digital media such as text, image, audio, video, etc. In the meantime, it is easier to obtain illegally acquisition, misappropriate and tamper with digital media. Therefore, the research on digital watermark technology has its significance for digital media protection. There are varieties of classification methods for digital watermarking. According to the anti-attack capability of watermark, digital watermark can be divided into robust watermark and fragile watermark. Robust watermark has strong anti-attack ability, and can remain intact or be accurately identified after undergoing various unintentional or intentional signal processing procedures. These signal processing processes include noise, filtering, digital/analog and analog/digital conversion, resampling, cropping, translation, rotation, scale change and compression coding, or malicious spoofing attacks such as 
forgery, illegal copying, etc. Robust watermarking is the focus of watermark research. It is mainly used to identify copyright information in digital works, such as author information and product serial number. Fragile watermark is sensitive to signal processing which can be used to determine whether the image is tampered, it is mainly used for integrity authentication. Watermarking algorithms can also be divided into blind watermarking and non-blind watermarking according to whether original signal is required for watermark detection or not. Non-blind watermark requires the original signal data during the detection process, whereas blind watermark detection does not. Therefore, blind watermarking is more widely used.

The print process is commonly used in image reconstruction and distribution. How to use digital watermarking technology in printed images is also a hot topic. This article presents a print-recapture robust watermark scheme in which watermark can be detected only using a smartphone. First, embed the watermark into an image or document, and then print the watermarked image or a document. Thereafter, invoke the detection application on a smartphone which equipped with a camera and aim it at the paper image or document so the watermark can be detected. After detection, perform authorization or connect to remote services, etc.

Some application scenarios of the proposed scheme are as follows. For example, the printrecaptured watermark scheme can be used for promotion in a supermarket. If there is watermark in the promotional posters, customers who are attracted by the products can get more products and purchase information by scanning the poster. And book readers can scan the pictures in the book to get the electronic version of the book then offline reading is transferred to online. Some other authorization operations can also be achieved through a watermarked image. In the watermark scheme, we use a spatial domain embedding method, and to ensure anywhere of the printed image can be detected and to make the synchronization, the watermark was embedded periodically.

The following is the arrangement of the remaining parts. The second part reviews related work. In the third part of the paper we make a detailed description of proposed method including embedding and extraction process as well as the synchronization principle. The fourth part is the experiment. The last part is the conclusion of the paper.

\section{RELATED WORK}

This chapter introduces related work from two aspects. First review some papers about print-scan watermark scheme. Then make a description of several watermark synchronization schemes that are common used.

\subsection{Print-Scan Process}

Watermark robust to print-scan process appears earlier than that in print-recapture system. Prof. ShihFu Chang and Ching-Yung Lin of Taipei, Taiwan, China, had modeled the process of print-scan in 1999 (Lin, 1999). The author proposed that print-and-scan causes pixel and geometric distortion for the image, they also extracted invariant through print-and-scan process. Longjiang Yu et al. made a description on printer and scanner used in print and scan progress in (Yu, 2005). In 2003, a print-andscan resilient watermark method was used for card authentication (Anthony, 2003). In paper (Solanki, 2006) from Kaushal Solanki et al. in 2006, the differences between printed image and the original image are coefficients of magnitude spectrum and phase of frequency domain. In 2008, Daofang Cheng et al. use statistics-based method in print-and-scan watermarking scheme (Cheng, 2008). With the rapid spread of mobile devices, some researchers hope to use mobile devices to detect watermarks. Compared to print-and-scan progress, the distortion of print-recapture process is worse. In 2008, the distortion of print-cam is analyzed in (Pramila, 2008). In 2013, Takaaki Yamada et al. proposed a method for detecting watermarks in print using smartphone by finding no mark area (Yamada, 2013). 


\subsection{Watermark Synchronization}

The traditional watermarking scheme, whether it is frequency or spatial domain scheme, is difficult to extract the watermark correctly if loss of synchronization after geometric attacks, especially cropping, scaling, and local distortion attacks which are important distortions in the process of printing and recapturing. In order to detect the watermark in the recaptured image, watermark synchronization must be performed in the first step.

For the synchronization of watermark, there are mainly three solutions. The geometric boundary correction method is detecting image boundaries. This method is mainly used when the image has an obvious geometric edge. The boundary of the image is obtained by linear detection or other methods, and then geometric transformation is inverted (Anthony, 2003; Yu, 2005; Pramila, 2008). Another method is to embed watermark as well as a template which can be used to invert geometric transformation. For example embed a template in frequency domain or embed periodic watermark in space domain directly (Pereira, 2000). Full-search method for geometrical correction can also achieve geometric robust watermark (Yamada, 2013). That is making assumptions of a combination of geometric transformations and inverting geometric transformations until watermark is detected.

\section{DESCRIPTION OF PROPOSED SCHEME}

The printed watermarked image can act as a link if more about the image such as content, source or author is wanted by the image reader. The application scenario of proposed method is shown in Figure 1. Watermark is embedded into a host image and a watermarked image is obtained. Then the watermarked image is printed using a color printer. The readers can hold a smart phone above the paper and invoke the watermark detection application if they want to know more about the image. The detection application is running until the watermark is detected. A relevant web is opened when the watermark is detected.

\subsection{Characteristics of Recaptured Images}

In paper (Lin, 1999), the distortion of print-scan was modeled from both pixel and geometric transformation, and the distortion of the print-scan process image was analyzed from frequency domain. Article (Pramila, 2008) analyzed print-cam process which causes barrel or pincushion distortions as well as distortion of print-scan. The distortion of recapturing by a smart phone is close to print-scan, but it is not exactly the same. First of all, the recaptured picture may be part of the whole image, and different part of the image is obtained when the phone is moved, which corresponds to the image after cropping and translation. Secondly, the scaling factor of the recaptured image is variable, which is determined by the distance between the phone and the image. The smaller the distance, the

Figure 1. Description of application scenario

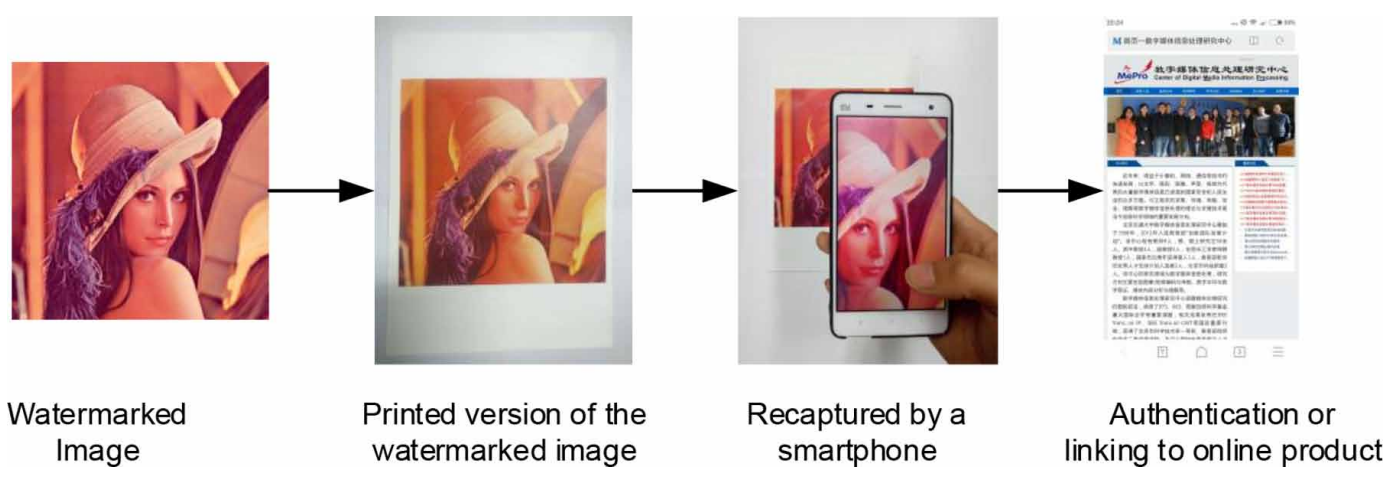


larger the image is scaled. Thirdly, the phone is not parallel to the recaptured image, which results in different scaling factors in different areas of the image. Lastly, assume that the phone is parallel to the image, rotating the phone horizontally is equivalent to image rotating. In addition, the handheld camera shakes slightly which blurs the image and the lighting of the environment also affects the quality of captured image, etc.

\subsection{Embedding Principle}

\subsubsection{Design of Watermark Pattern}

In order to locate (or synchronize) the watermark pattern $\mathrm{W}$ accurately, the pattern must be a centrosymmetric graphic which means that the pattern looks same when it is rotated at an angle of 180 degrees. Since the angle of the mobile phone is arbitrary and to reduce computational complexity during the process of watermark detection, the pattern $\mathrm{W}$ when rotated by the angle of 90,180 and 270 degrees appears same as before. By embedding watermark pattern periodically, it can be detected in a cropped image.

\subsubsection{Watermark Embedding}

Figure 2 is the chart flow of watermark embedding. First a host RGB color image is obtained. Then the host image is converted to CIE Lab color space and its Lab image $I_{L a b}$ is obtained. And watermark pattern $W$ is periodically extended to get periodic watermark $W_{\text {peri }}$. In order to detect watermark effectively, $W_{\text {peri }}$ is embedded in both $a$ and $b$ color channels of the Lab image. Embed the watermark $W_{\text {peri }}$ into color channel $a$ according to formula (1).

$$
I_{a}^{\prime}(i, j)=I_{a}(i, j)+\alpha \cdot N V F(i, j) \cdot W_{p e r i}(i, j)+\beta \cdot(1-N V F(i, j)) \cdot W_{p e r i}(i, j)
$$

Wherein, $I_{a}(i, j)$ is $a$ color channel of $I_{L a b}$ and $I_{a}^{\prime}(i, j)$ is $I_{a}(i, j)$ after modification, $\alpha$ and $\beta$ are constants which control the strength of the watermark in smooth and texture area separately, and $N V F$ stands for Noise Visibility Function of $I_{a}, N V F(i, j)$ shows local texture change of band $L L$ at coefficient position $(i, j)$ and ranges among $(0,1)$, when local texture is in extreme flat, $N V F$ verges to 1 , contrarily, verges to 0 . Visit the web site for more details about $N V F$ : https://in.mathworks. com/matlabcentral/answers/89979-how-to-make-noise-visibility-funtion.html.

Figure 2. Flowchart of watermark embedding

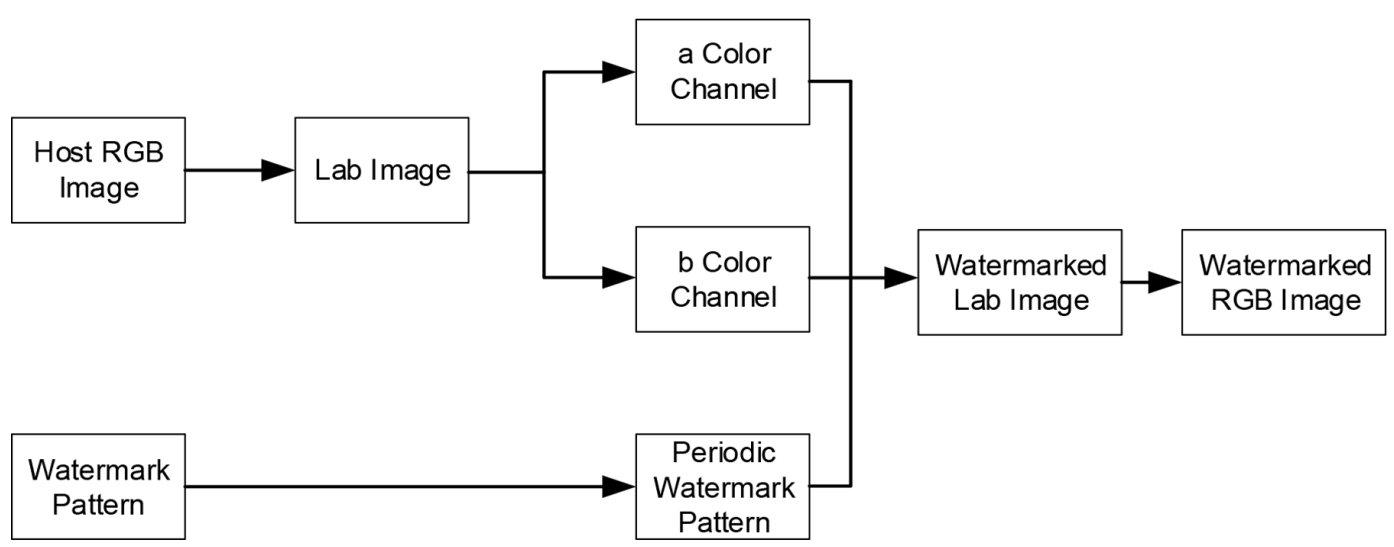


For $b$ color channel, use $\tilde{W}_{\text {peri }}=255-W_{\text {peri }}$ instead of $W_{\text {peri }}$ in formula (1), and use new weight matrix by calculating $N V F$ of $I_{b}$, do the same as a color channel and get $I_{b}^{\prime}(i, j)$. Merge $I_{L}(i, j)$, $I_{a}^{\prime}(i, j)$, and $I_{b}^{\prime}(i, j)$ to get watermarked Lab image $I_{L a b}^{\prime}$. Then get watermarked RGB image $I_{R G B}^{\prime}$ by converting $I_{L a b}^{\prime}$ from Lab to RGB color space. Print $I_{R G B}^{\prime}$ on a paper or display it on a screen and a smart phone with a certain program can be used to detect the watermark as illustrated in Figure 1.

\subsection{Extraction Principle}

\subsubsection{Traditional Solution by Calculating ACF}

Auto Correlation Function $(A C F)$ is regularly used in watermark synchronization scheme (Kutter, 1999). Calculate $A C F$ for periodic images to produce local peaks which can be used to invert geometric transformation. The basic principle is that a random matrix has high correlation with itself and low correlation with other random matrices. In detail, Figure 3 can be regarded as a random matrix, and Figure 4 is a periodic matrix of Figure 3. Cut Figure 4 in four directions and the part inside the box is left. Calculate $A C F$ for the left part using formula (2).

$$
A C F_{D}(s, t)=\sum_{m=0}^{M-1} \sum_{n=0}^{N-1} D(m, n) D(m+s, n+t)
$$

if $0 \leq s<2 M-1$ and $0 \leq t<2 N-1$

Wherein, $M$ and $N$ are the number of rows and columns of matrix $D$. During the calculation, the top left $P_{1}^{\prime}$ and bottom right image $P_{1}$ are the same in Figure 5(a). In the process of calculation, image $P_{1}^{\prime}$ slides over image $P_{1}$. When $P_{1}^{\prime}$ is moved to the position as Figure 5(a) and 5(b), the result equals to calculate the autocorrelation value of the part inside of the box, which is much larger than other locations (shown in Figure 6). So, in such places there are local peaks which can be used to synchronize watermark.

But if we want to locate a watermark pattern (Figure 3) from the cropped periodic watermark pattern, ACF doesn't work. For example, if the size of the pattern in Figure 3 is $m \times m$, and $C_{1}, C_{2}$, $C_{3}, C_{4}$ pixels is cropped in the top, bottom, left and right direction respectively. The coordinates of local peaks are $\left(k_{1} m-C_{3}-C_{4}, k_{2} m-C_{1}-C_{2}\right)$, wherein $k_{1}, k_{2} \in R$. And the position of the first peak is $\left(m-C_{3}-C_{4}, m-C_{1}-C_{2}\right)$. That is the summarization of the two widths of the remaining two incomplete blocks after left and right cropping and the summarization of the two heights of the remaining two incomplete blocks after top and bottom cropping. Therefore, $C_{1}, C_{2}, C_{3}$ or $C_{4}$ can't be determined and that is to say it is unknown that how much of the figure is cropped in four directions respectively. The conclusion is that when a watermarked image is cropped, the embedded periodic pattern is also cropped correspondingly, peaks in ACF can't help to synchronize.

\subsubsection{Proposed Scheme by Calculating Self-Convolution}

When the pattern in Figure 3 is replaced by a centrosymmetric pattern which is the same as itself when rotated 180 degrees shown in Figure 7, do the same as above and get Figure 8. Calculate selfconvolution instead of ACF according to equation (3). During the calculation, the top left $P_{2}^{\prime}$ is the bottom right image $P_{2}$ after rotating 180 degrees in Figure 9(a). The top left image $P_{2}^{\prime}$ slides over $P_{2}$. When $P_{2}^{\prime}$ is moved to the position as Figure 9(a) and 9(b), the result equals to calculate the 


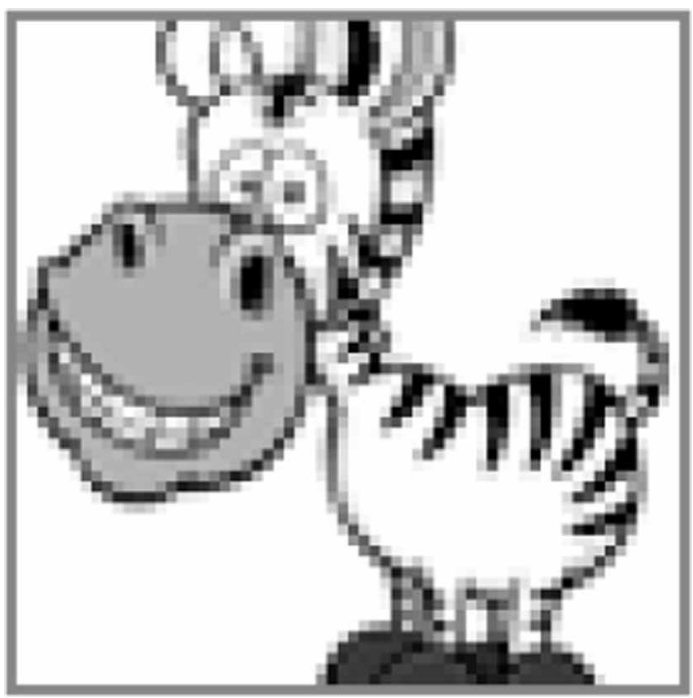

autocorrelation value of the part inside of the box, which is much larger than other locations. So in such places there are local peaks which can be used to synchronize watermark.

And if we want to locate a watermark pattern (Figure 7) from the cropped periodic watermark, self-convolution can be used. For example, if the size of the pattern in Figure 7 is $m \times m$, and we cut $C_{1}, C_{2}, C_{3}, C_{4}$ pixels in the top, bottom, left and right directions respectively.

The coordinates of local peaks are $\left(k_{1} m-2 C_{1}, k_{2} m-2 C_{3}\right)$, wherein $k_{1}, k_{2} \in R$. And the position of the first peak is $\left(m-2 C_{1}, m-2 C_{3}\right)$, as well as $C_{2}$ and $C_{4}$ are obtained, and watermark can be synchronized after cropping.

$\operatorname{SConv}_{D}(s, t)=\sum_{m=0}^{M-1} \sum_{n=0}^{N-1} D(m, n) D(s-m, t-n)$

if $0 \leq s<2 M-1$ and $0 \leq t<2 N-1$

Wherein, $M$ and $N$ are the number of rows and columns of matrix $D$.

\subsubsection{Watermark Extraction}

Figure 10 is the chart flow of watermark extraction. The detection process can be described as the following three steps. First, reduce the size of the recaptured image. Cut the recaptured image $\hat{I}$ and remain the center of it and use methods such as the interpolation to shrink the image to half its length and width to reduce computation because full analysis is too complex and unnecessary. Then convert it from RGB to Lab to get the estimation of watermark $\hat{W}_{\text {peri }}$ which must contain at least more than one watermark pattern. $\hat{W}_{\text {peri }}$ is calculated using formula (4).

$\hat{W}_{\text {peri }}=\hat{I}_{a}+\hat{I}_{b}$ 


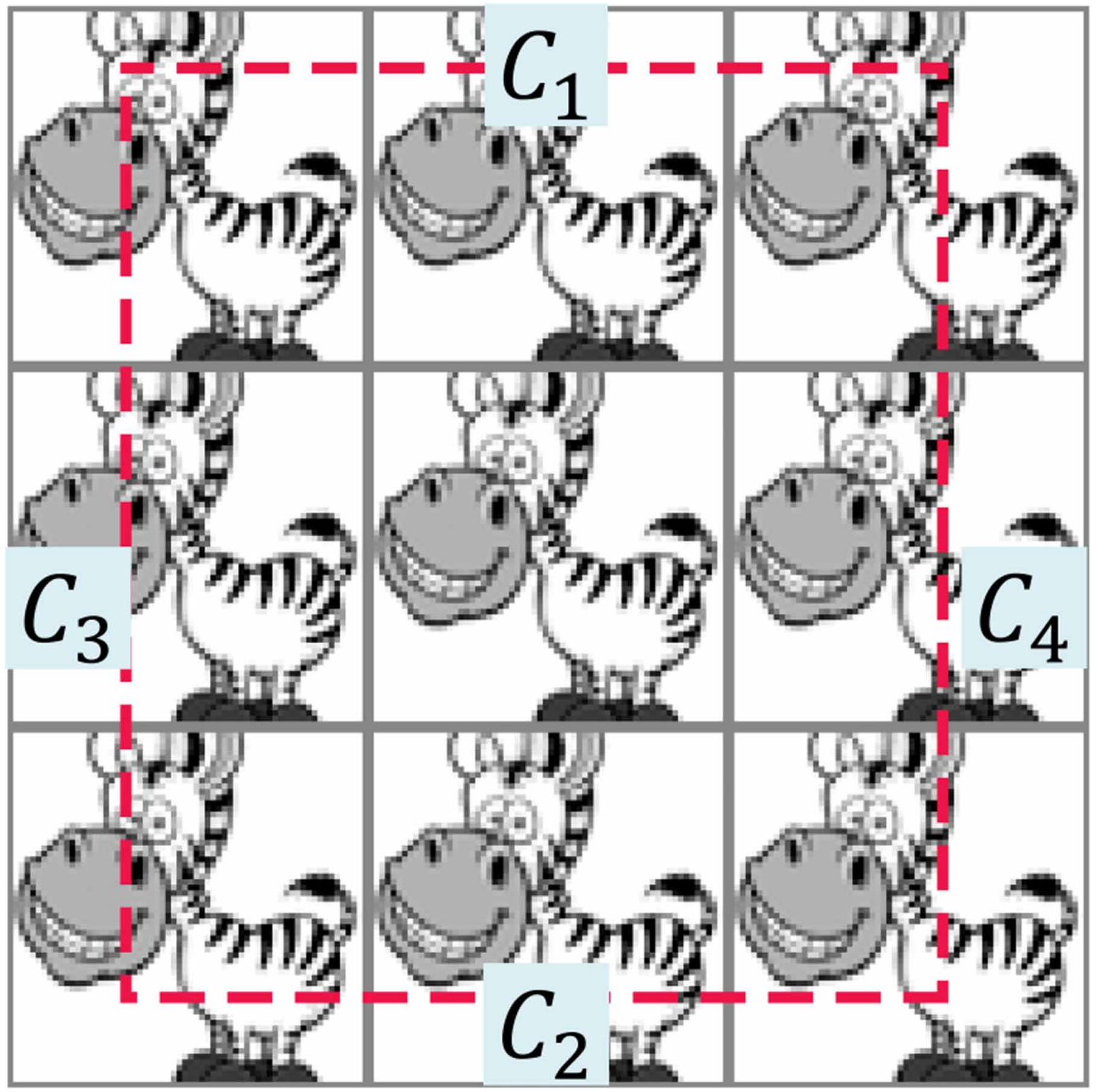

Wherein, $\hat{I}_{a}$ and $\hat{I}_{b}$ are $a$ and $b$ color channels of the recaptured image $\hat{I}$.

Second, locate one watermark pattern $W^{\prime}$ from $\hat{W}_{\text {peri }}$, also called watermark synchronization. For the watermark pattern is embedded periodically, $\hat{W}_{\text {peri }}$ also has periodicity. Calculate selfconvolution of the periodic signal of a central symmetric base signal, and the base signal can be located according to the local peaks of the self-convolution function. Instead of calculating selfcorrelation (also called $A C F$ ) to invert scale and rotation but not location as done in (Kutter, 1999), from the self-convolution function, we can also locate watermark pattern as well as invert scale and rotation. The order is, according to the distribution of the peak, first correct the rotation, then the scale, and finally location a pattern.

Third, by comparing $W^{\prime}$ and the embedded watermark pattern $W$, whether watermark is contained or not can be judged. Use normalized correlation coefficients $(N C)$ to represent the similarity between $W^{\prime}$ and $W$. And the range of $N C$ is from -1 to 1 . 
Figure 5. The principle of local peaks generation In ACF
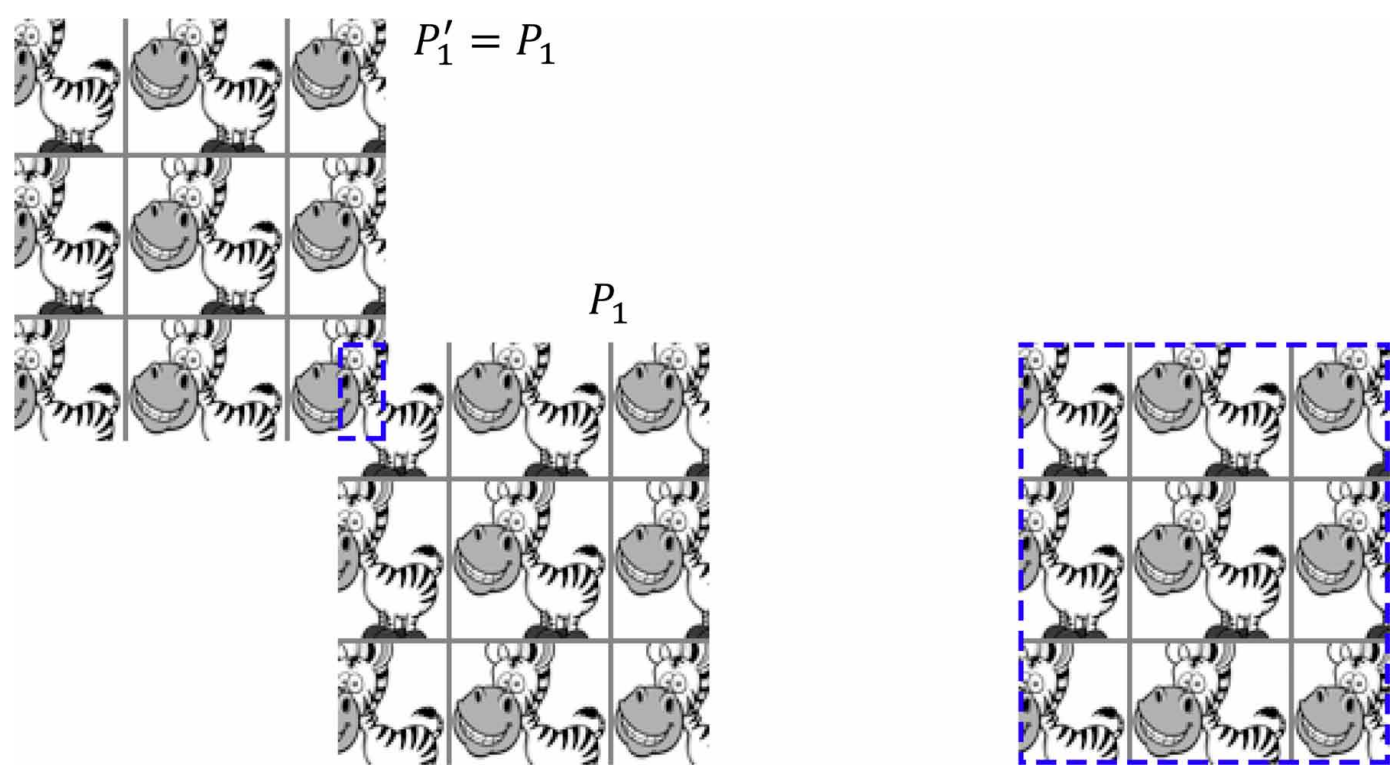

Figure 6. Local peaks generation In ACF

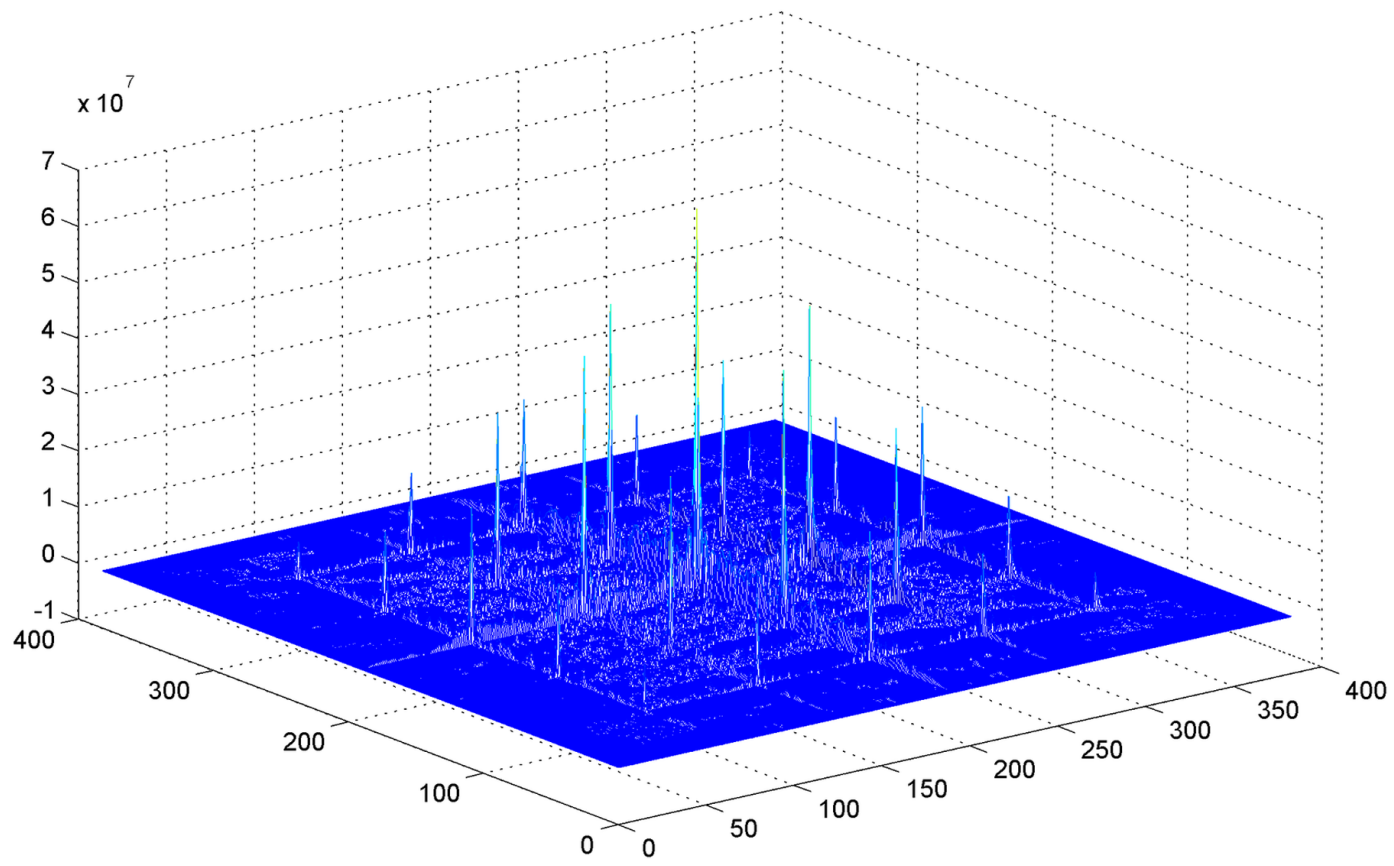


International Journal of Digital Crime and Forensics

Volume 11 • Issue 4 • October-December 2019

Figure 7. Centrosymmetric watermark pattern. It Looks the same when rotated at an angle of $\mathbf{1 8 0}$ degrees.

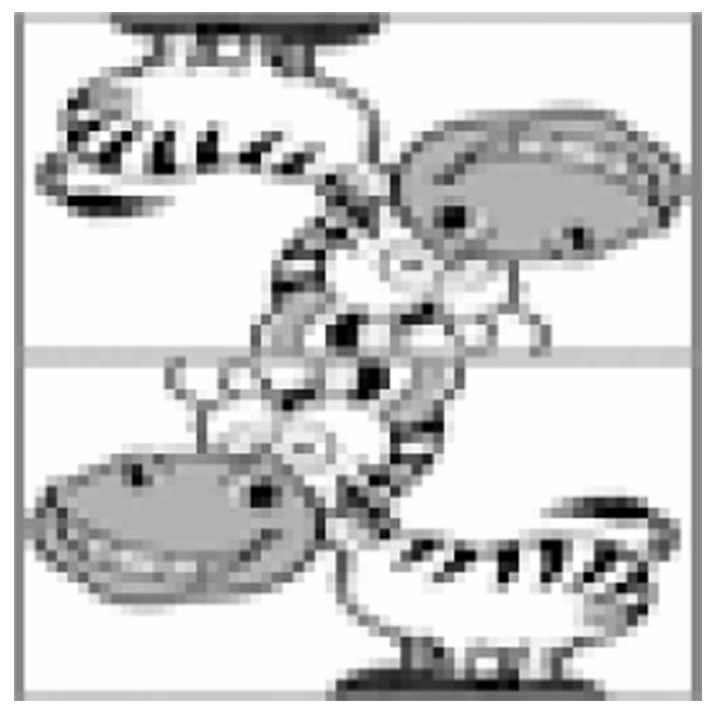

Figure 8. Periodic watermark pattern

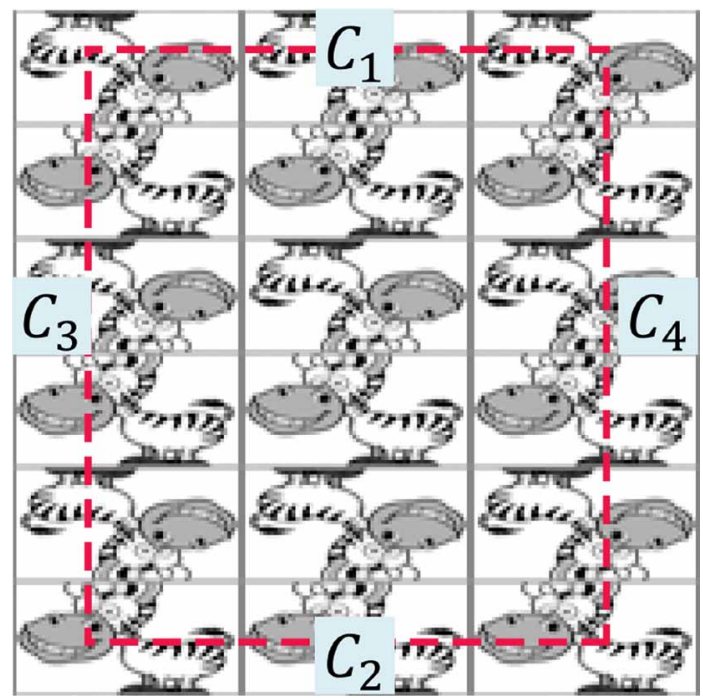


Figure 9. The principle of local peaks generation in self-convolution
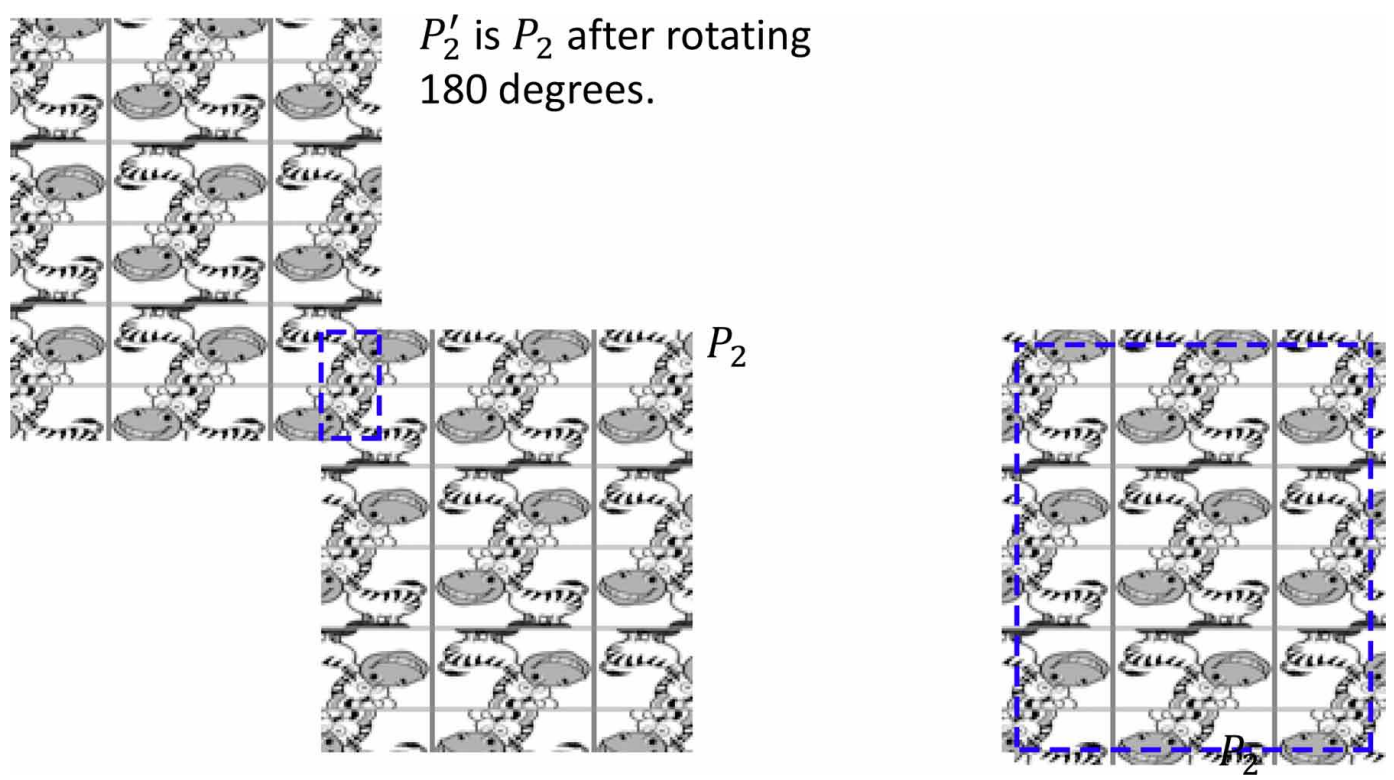

Figure 10. Flowchart of watermark extraction

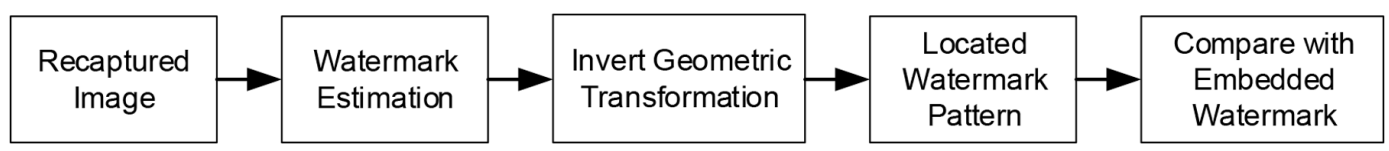

$$
N C\left(W, W^{\prime}\right)=\frac{\sum_{i=1}^{M} \sum_{j=1}^{N}[W(i, j)-\bar{W}]\left[W^{\prime}(i, j)-\overline{W^{\prime}}\right]}{\sqrt{\sum_{i=1}^{M} \sum_{j=1}^{N}[W(i, j)-\bar{W}]^{2}} \sqrt{\sum_{i=1}^{M} \sum_{j=1}^{N}\left[W^{\prime}(i, j)-\overline{W^{\prime}}\right]^{2}}}
$$

Wherein, $\bar{W}=\frac{1}{M N} \sum_{i=1}^{M} \sum_{j=1}^{N} W(i, j)$, and $\overline{W^{\prime}}=\frac{1}{M N} \sum_{i=1}^{M} \sum_{j=1}^{N} \overline{W^{\prime}}(i, j)$.

\section{EXPERIMENT}

This part is mainly divided into two sections. Firstly, the watermark embedding is implemented and the watermarked image is shown. Secondly, two sets of recaptured images which include watermark images and images without watermark are selected for analysis. The similarity between the extracted watermark and embedded watermark is calculated using several methods. The best measurement is chosen and the threshold is given. 


\subsection{Watermark Embedding}

Embed the above watermark pattern shown in Figure 11 in different test images with different strength to obtain watermarked color images. Then the peak signal to noise ratio (PSNR) of the image before and after the embedding is calculated. Some of the watermarked images and their PSNR are shown in Figure 12a-12d. When the watermark is embedded, the values of color channel $a$ and color channel $b$ are increased, so theoretically the image becomes more reddish and yellowish. From the watermarked images, the visual effect also verifies this conjecture. After that the watermarked images are printed in color to obtain their paper version.

\subsubsection{Testing Images and Choosing Threshold}

The watermark detection application is installed on a smart phone. Then invoke the application and held the smart phone above the printed watermarked image to obtain a frame of image constantly. Once the watermark is detected, jump to a web page. The camera uses the automatic mode when acquiring images, and in order to ensure the image quality as much as possible, the illumination is sufficient. In order to find a suitable method for comparing the extracted watermark with embedded watermark and determine the appropriate threshold, 456 screenshot images were recaptured for the test, of which 241 were taken above watermarked images and 215 from normal images without watermark.

One of the images recaptured above watermarked image by a smart phone is shown in Figure 13. The distribution of NC, RHO, PSNR, and SSIM (Wang, 2004) between the extracted watermark and embedded watermark are scattered as shown in Figure 14a-14d. NC, RHO and PSNR are solved using formula (5), (6) and (7). And SSIM can be calculate with the method in (Wang, 2004). Watermark were extracted from the 456 images. For example, in Figure 14(a), horizontal axis is the index of pictures and vertical axis indicates $N C$ between extracted watermark and embedded watermark pattern.

$$
R H O=\frac{\sum_{i=1}^{M} \sum_{j=1}^{N} W_{i, j} W_{i, j}^{\prime}}{\sqrt{\sum_{i=1}^{M} \sum_{j=1}^{N}[W(i, j)]^{2}} \sqrt{\sum_{i=1}^{M} \sum_{j=1}^{N}\left[W^{\prime}(i, j)\right]^{2}}}
$$

$P S N R=10 \log _{10} \frac{255^{2}}{M S E}$

Wherein, $M S E=\frac{1}{M N} \sum_{i=1}^{M} \sum_{j=1}^{N}\left[W(i, j)-W^{\prime}(i, j)\right]^{2}$.

To make it clear, we draw a figure of false alarm rate and detection rate for the above four methods shown in Figure 15a-Figure 15d. From it we can see that the highest detection rate of the four methods is $0.83,0.33,0.35$, and 0.82 when the false alarm rate is zero. In order to achieve a better detection result, only $N C$ and SSIM can be used to distinguish whether there is watermark or not. Because the calculation complexity of SSIM is higher than $N C$, in our scheme we use $N C$ as correlation between extracted and embedded watermark. Select an appropriate threshold $N C_{T}$ to make the decision on whether there is watermark. For safety reasons, $N C_{T}$ must be set bigger than 0.09 shown in Figure 15(a). In our scheme, we chose 0.13 as $N C_{T}$, and the detection rate of 241 pictures is about 0.79 . 
Figure 11. Watermark pattern (W) used in the article

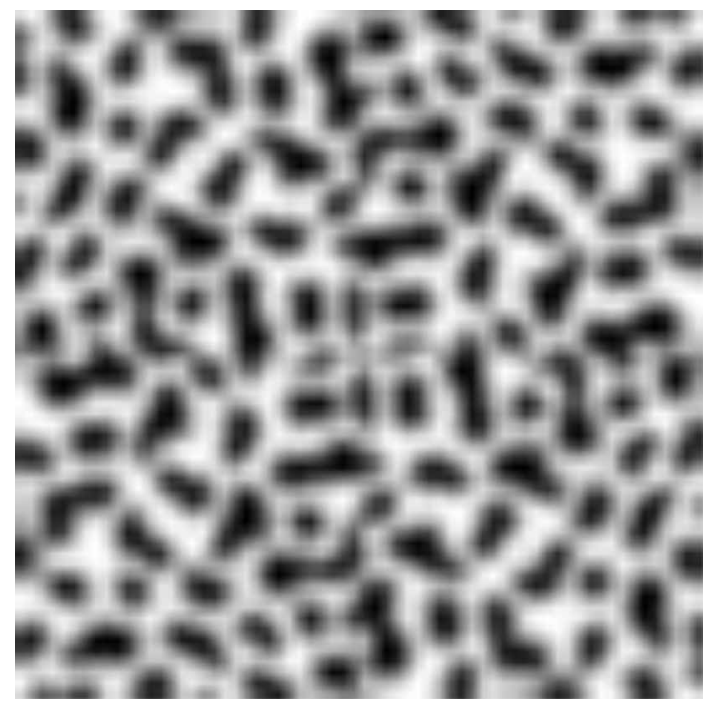

Figure 12a. Watermarked images and their PSNR

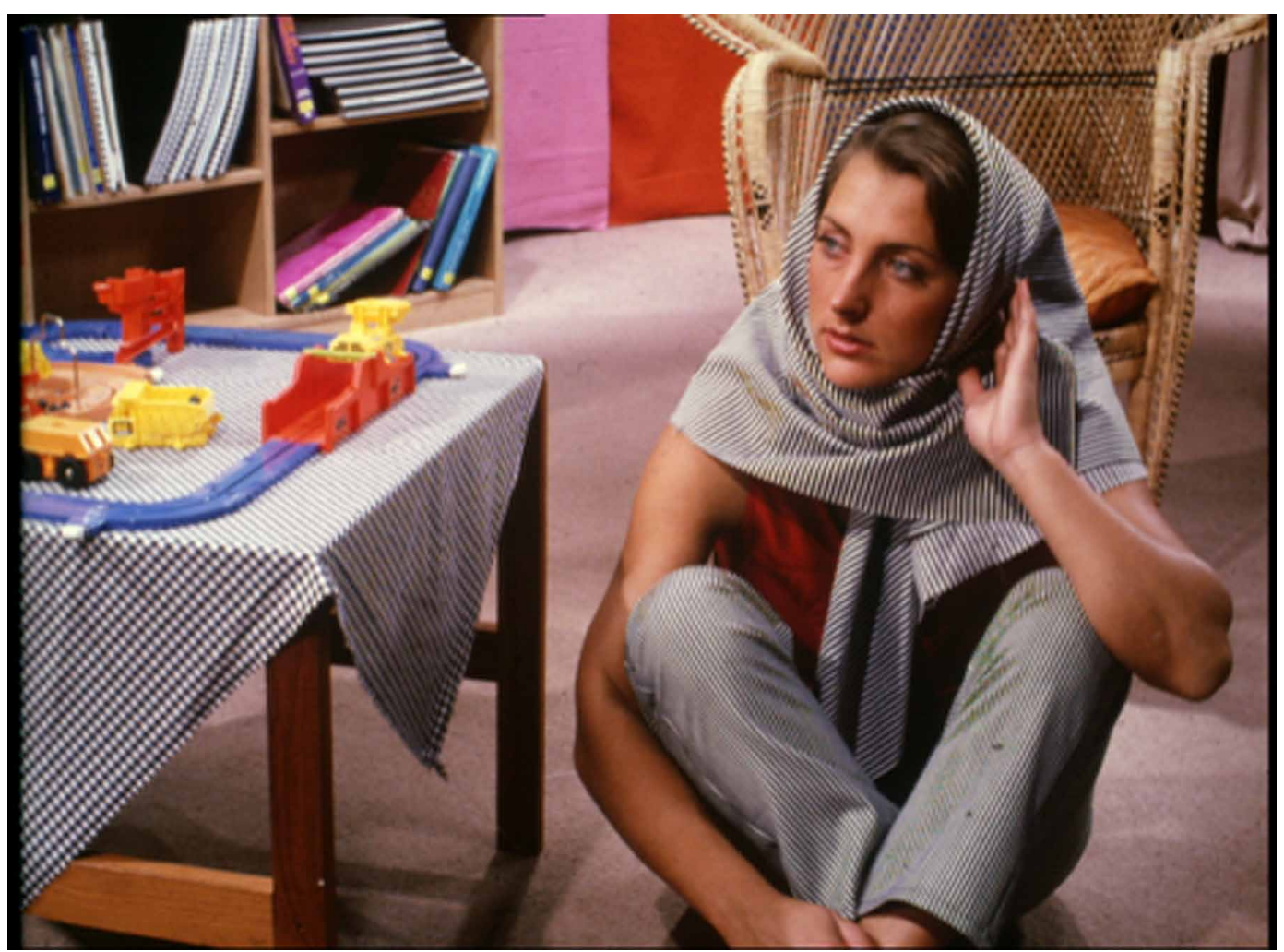


International Journal of Digital Crime and Forensics

Volume 11 • Issue 4 • October-December 2019

Figure 12b. Watermarked images and their PSNR

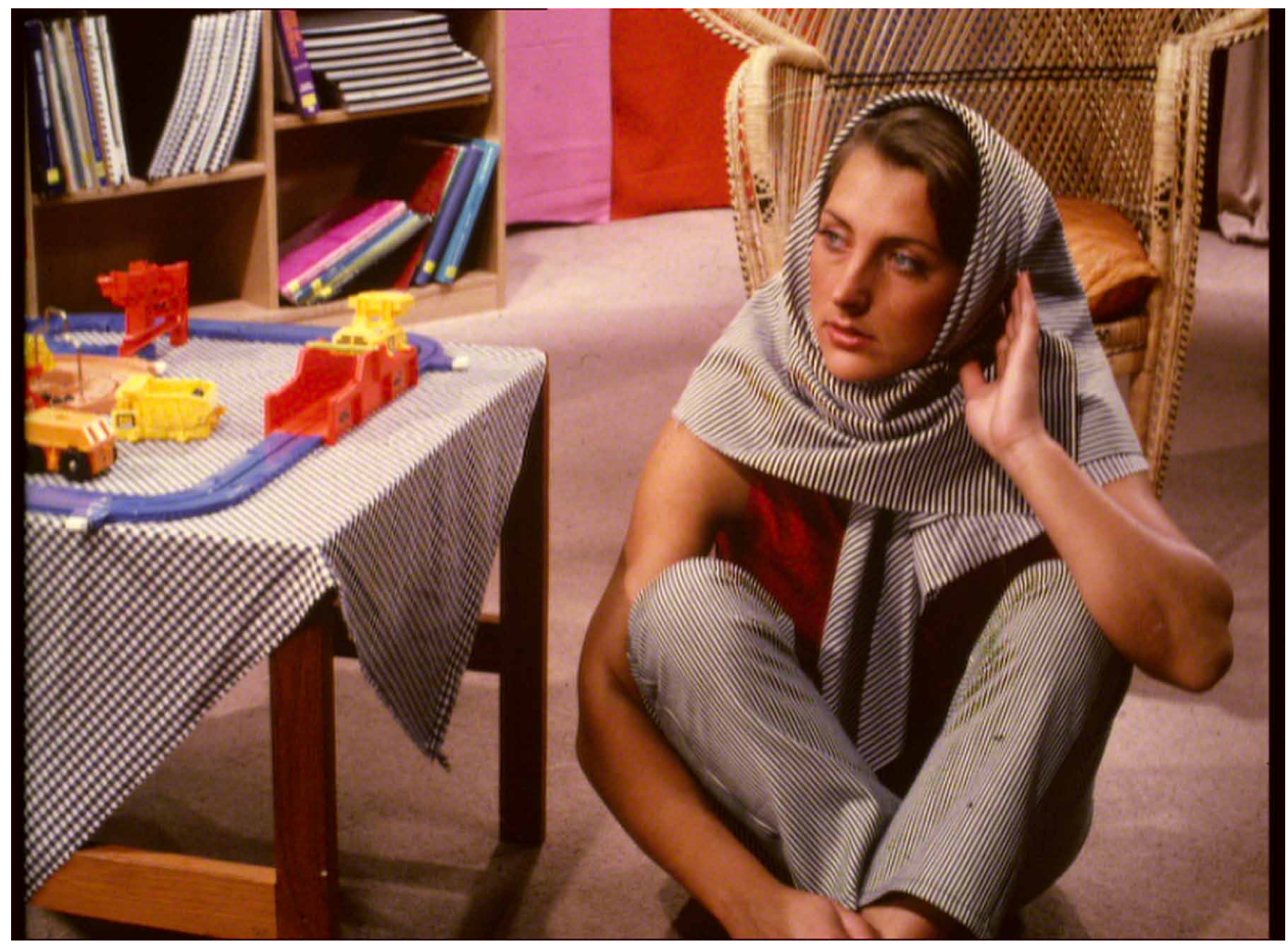


Figure 12c. Watermarked images and their PSNR

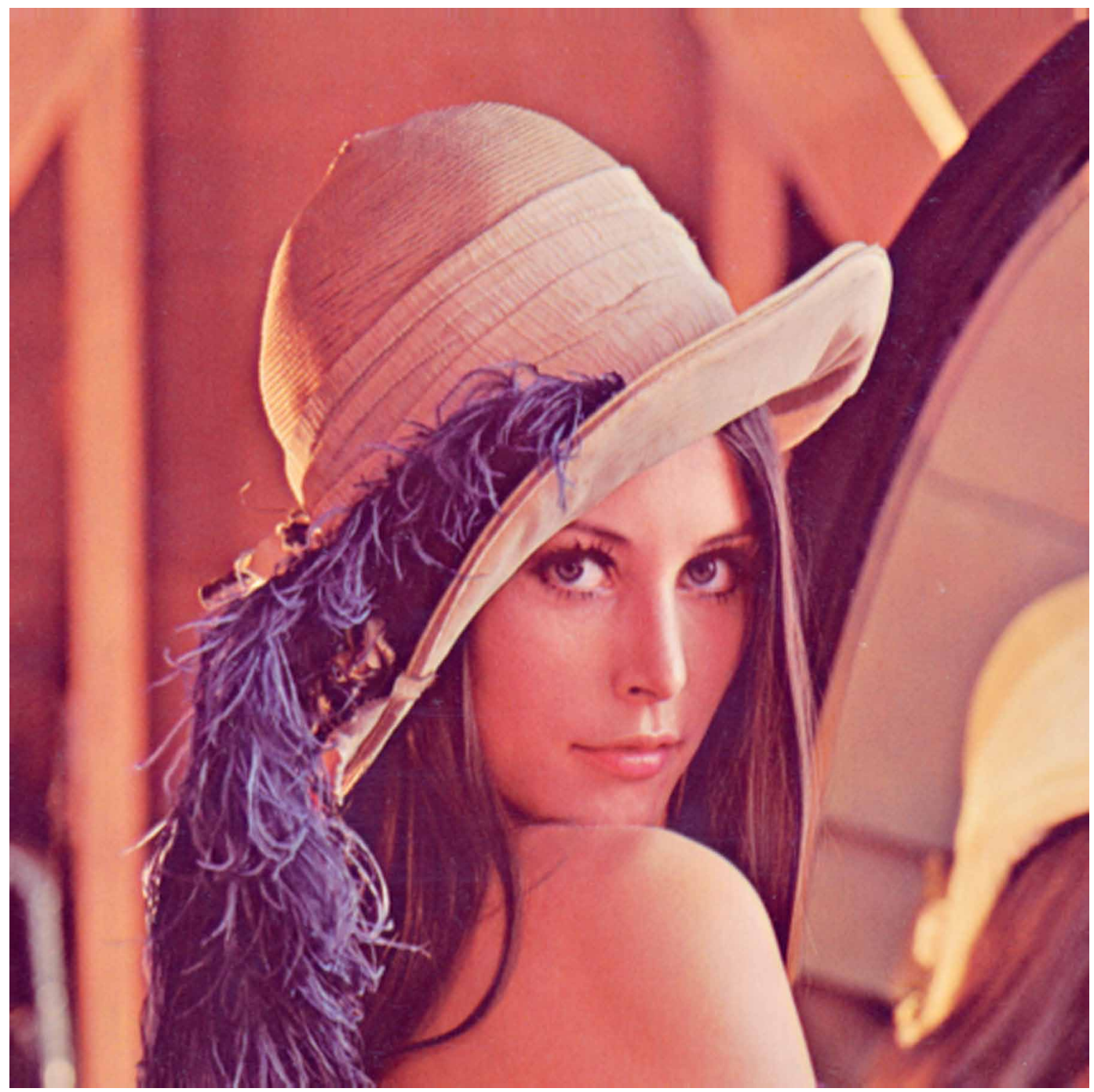


International Journal of Digital Crime and Forensics

Volume 11 • Issue 4 • October-December 2019

Figure 12d. Watermarked images and their PSNR

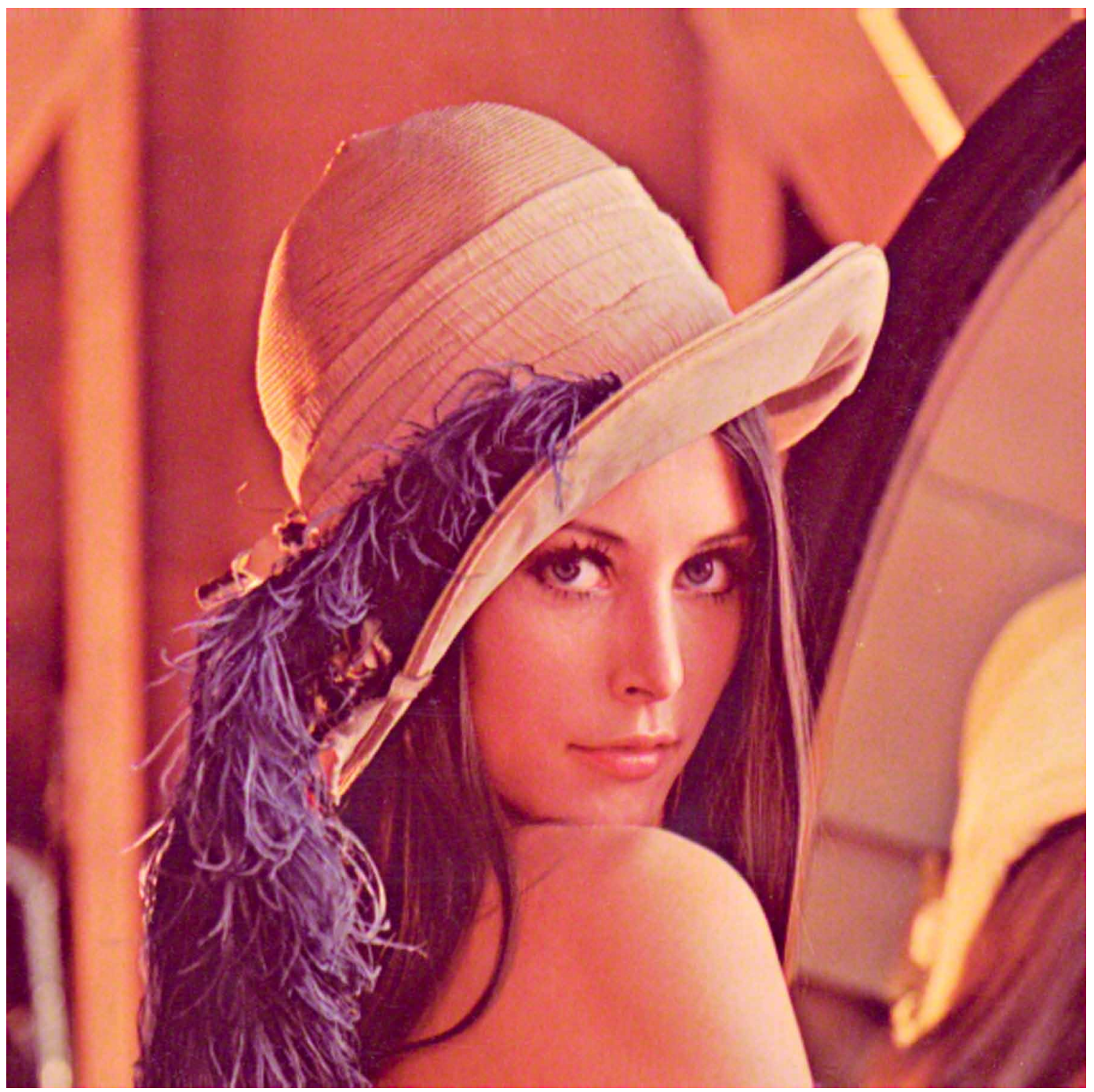




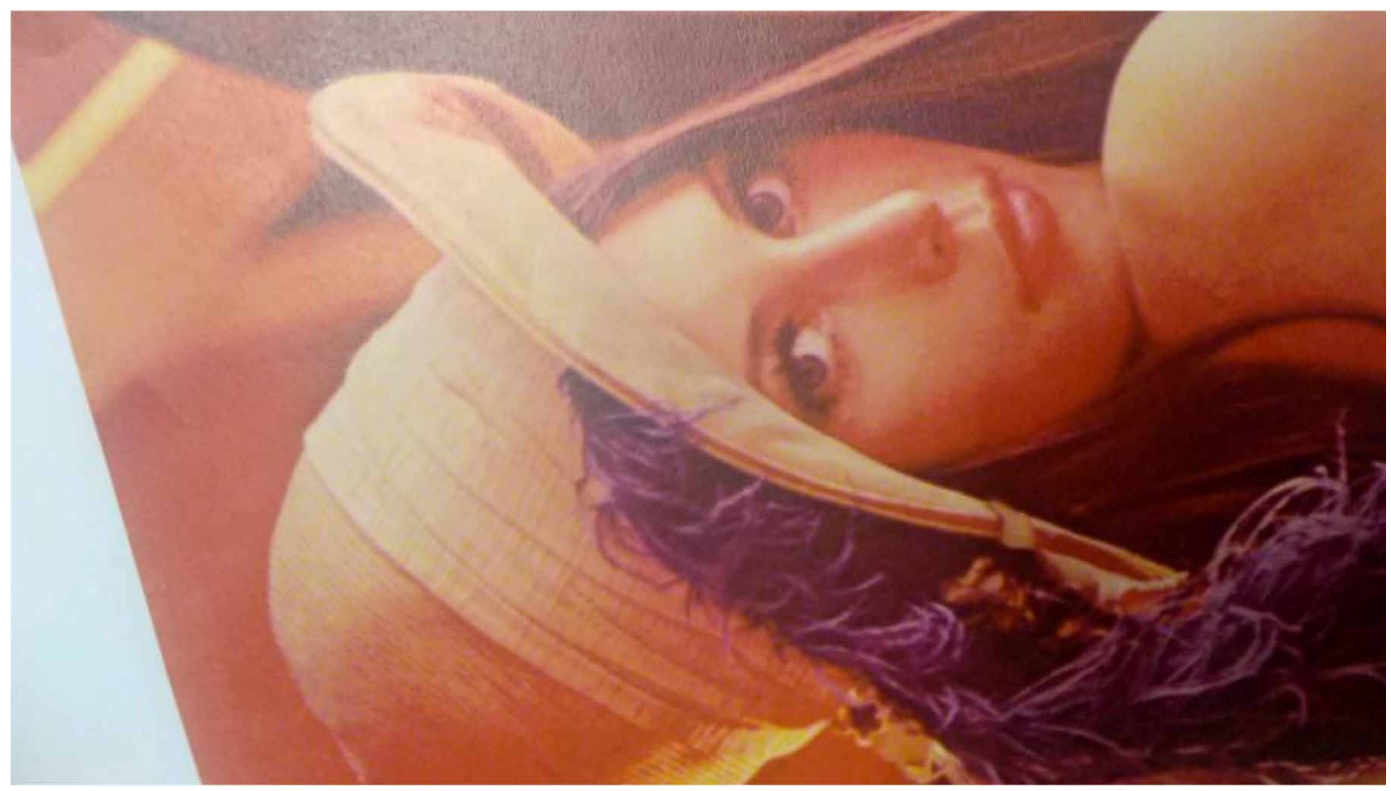

Figure 14a. Distribution of similarity parameters

comparison of $\mathrm{NC}$ of watermark images and images without watermark

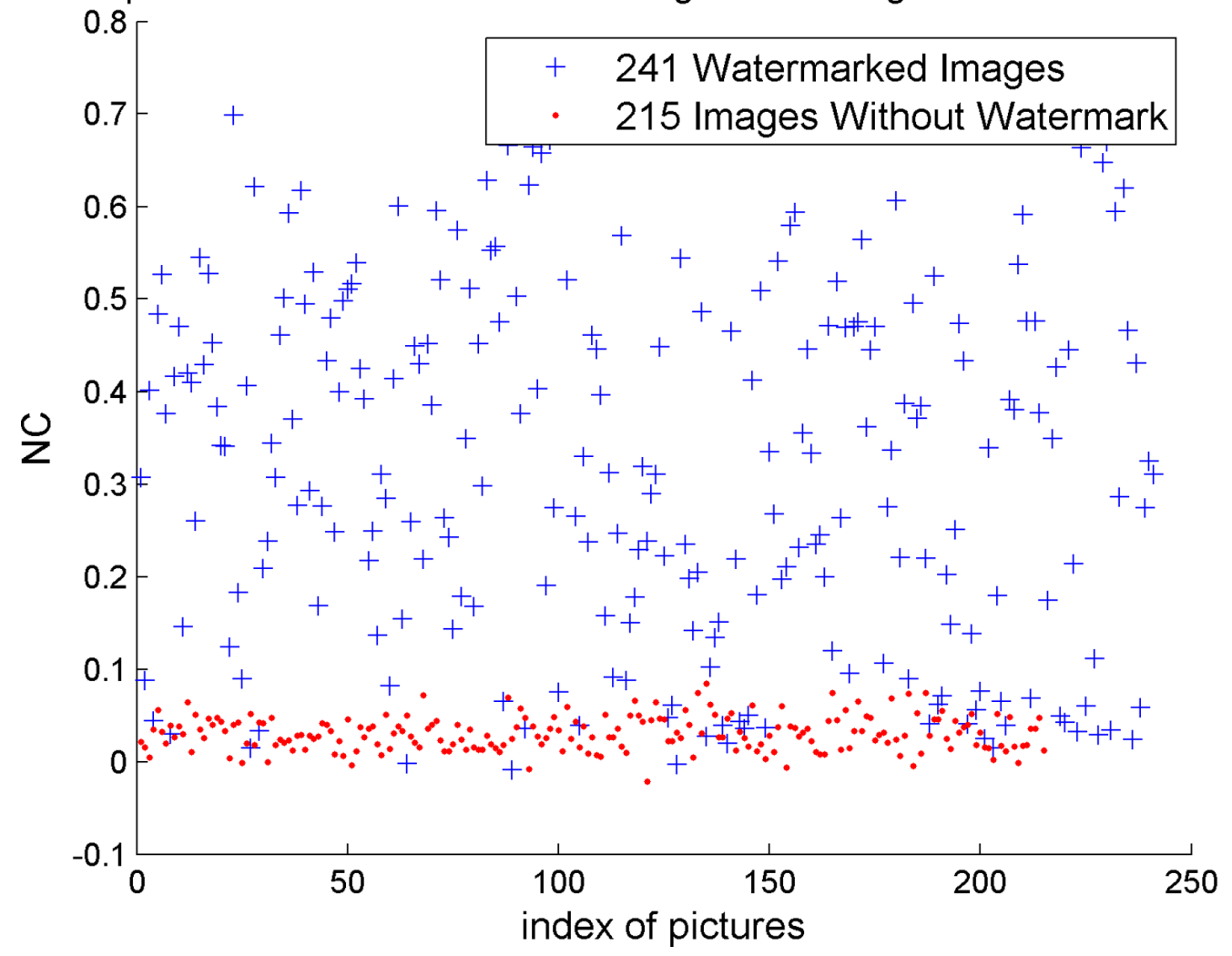


Figure 14b. Distribution of similarity parameters

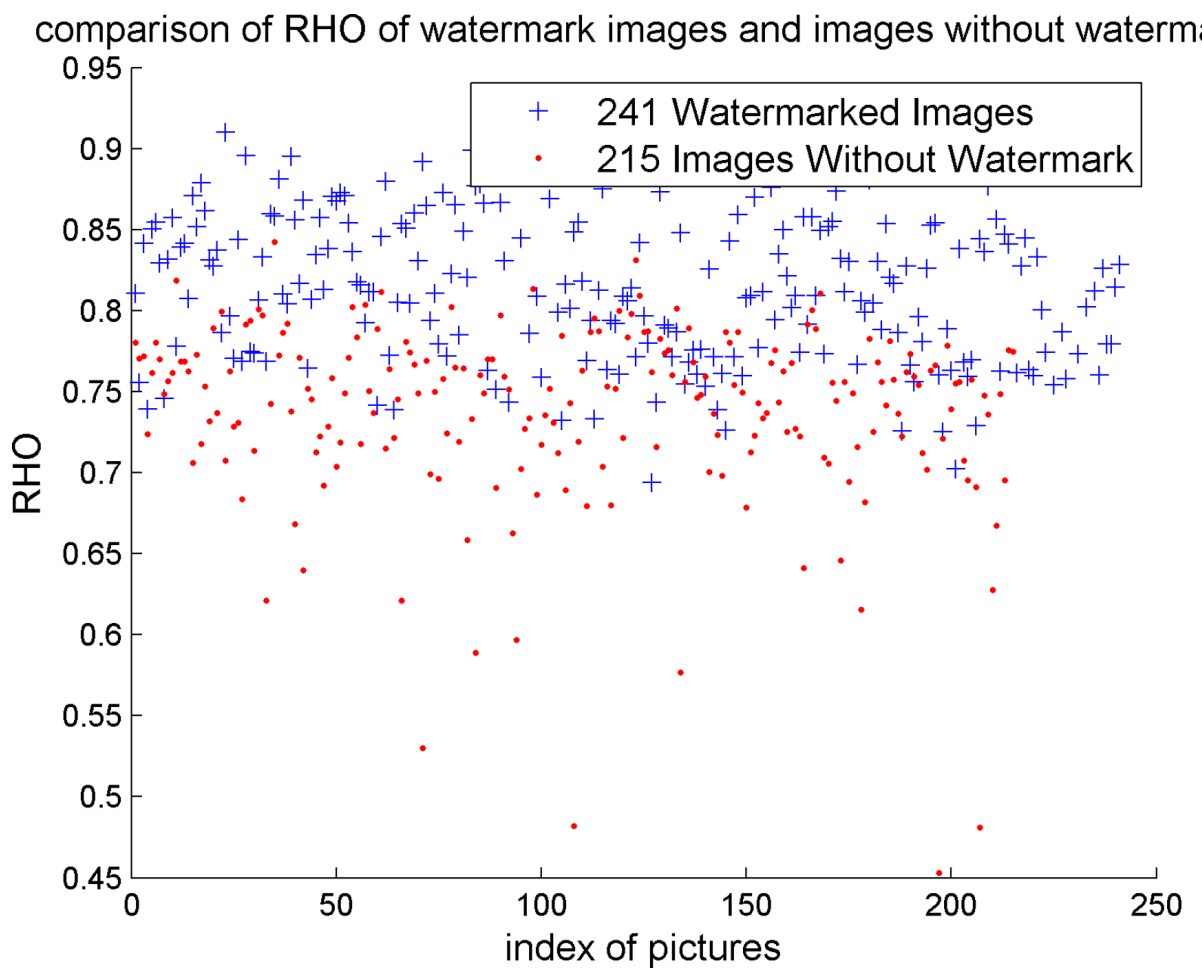

Figure 14c. Distribution of similarity parameters

comparison of PSNR of watermark images and images without watermark

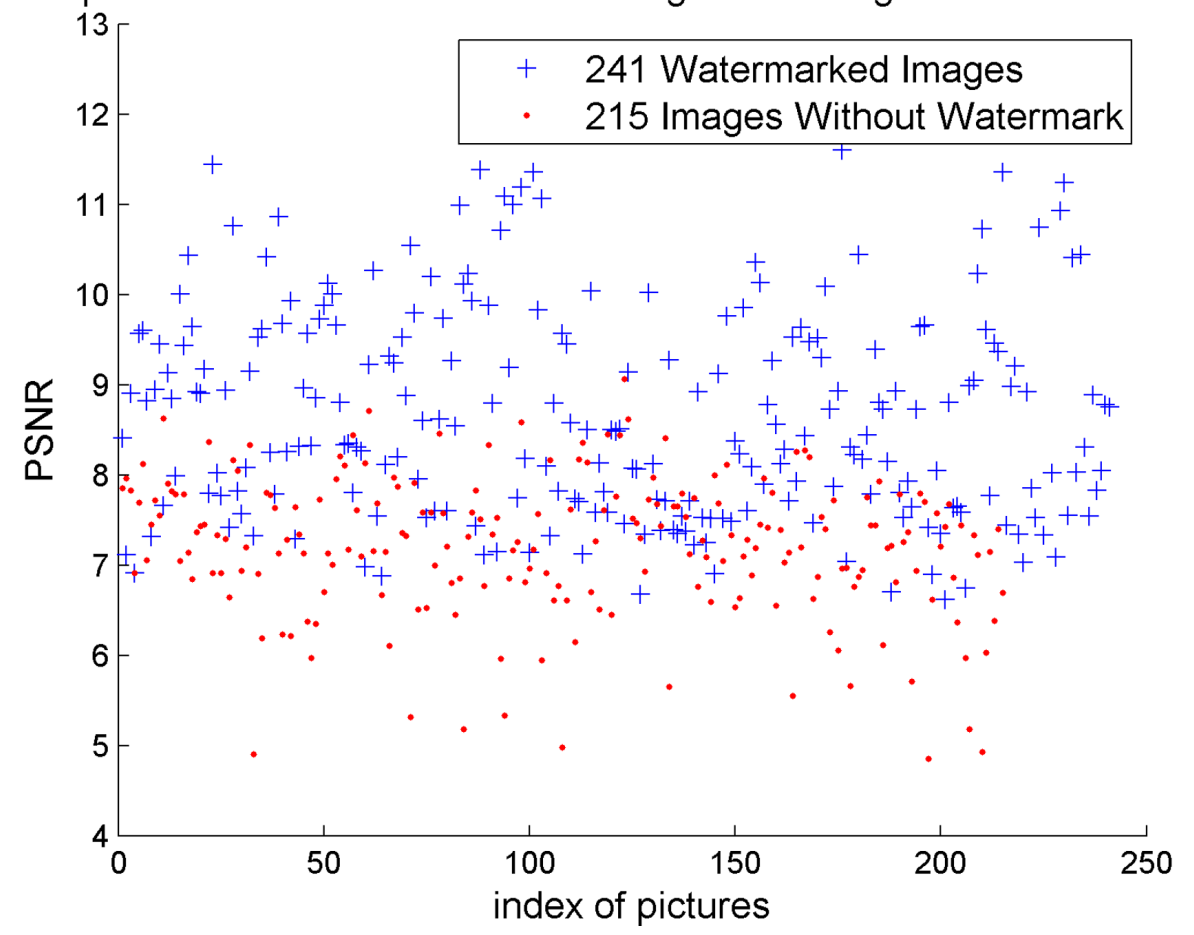


Figure 14d. Distribution of similarity parameters

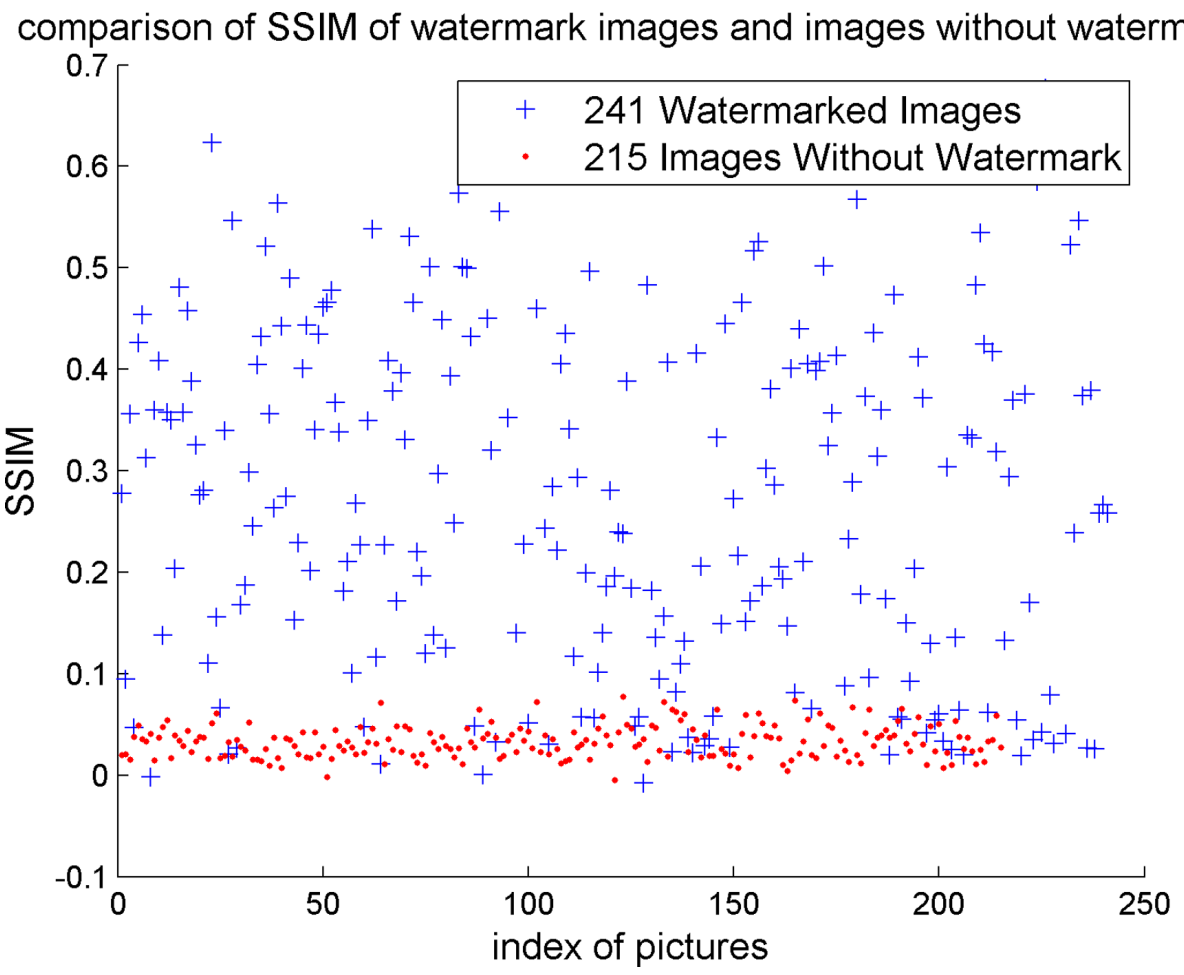

Figure 15a. Detection/false alarm rate of four methods. Point $P$ Is the highest detection rate when false alarm rate is zero.

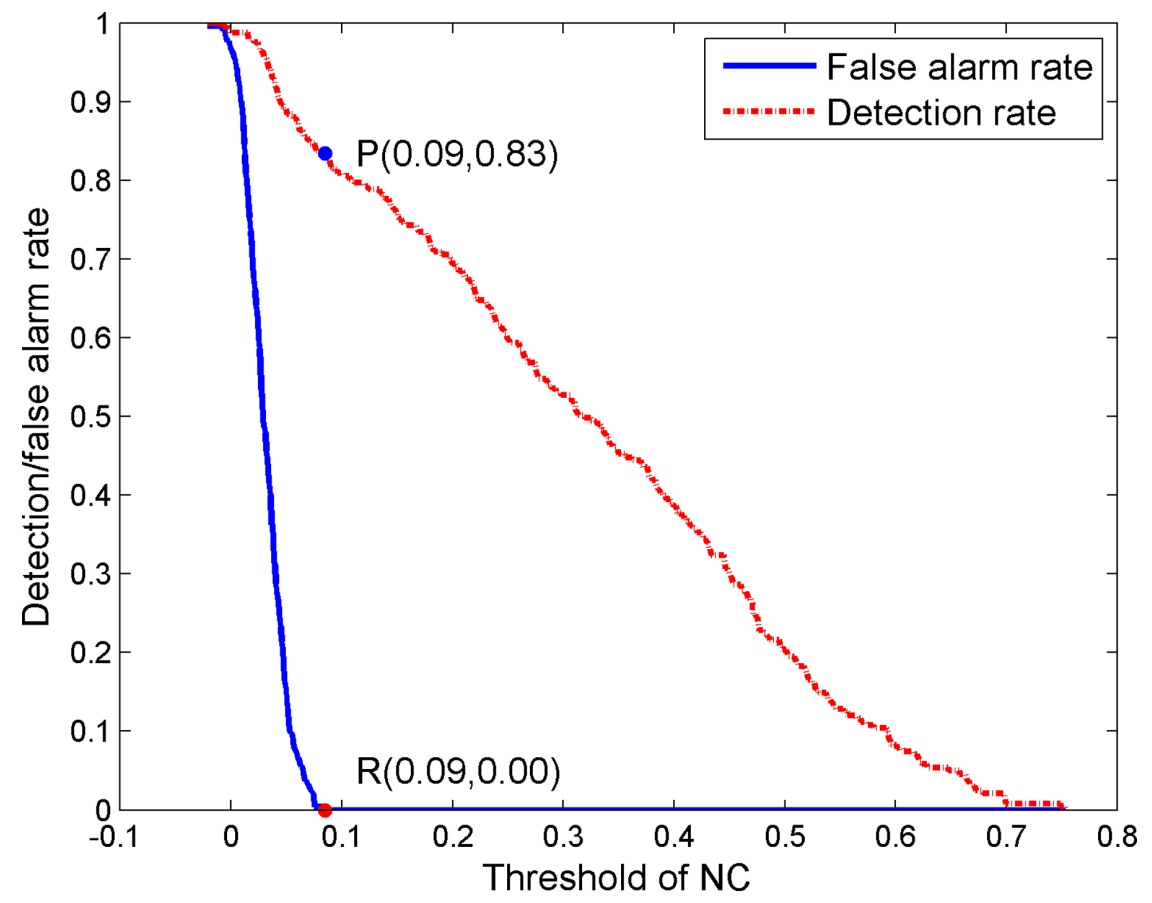


Figure 15b. Detection/false alarm rate of four methods. Point $P$ Is the highest detection rate when false alarm rate is zero.

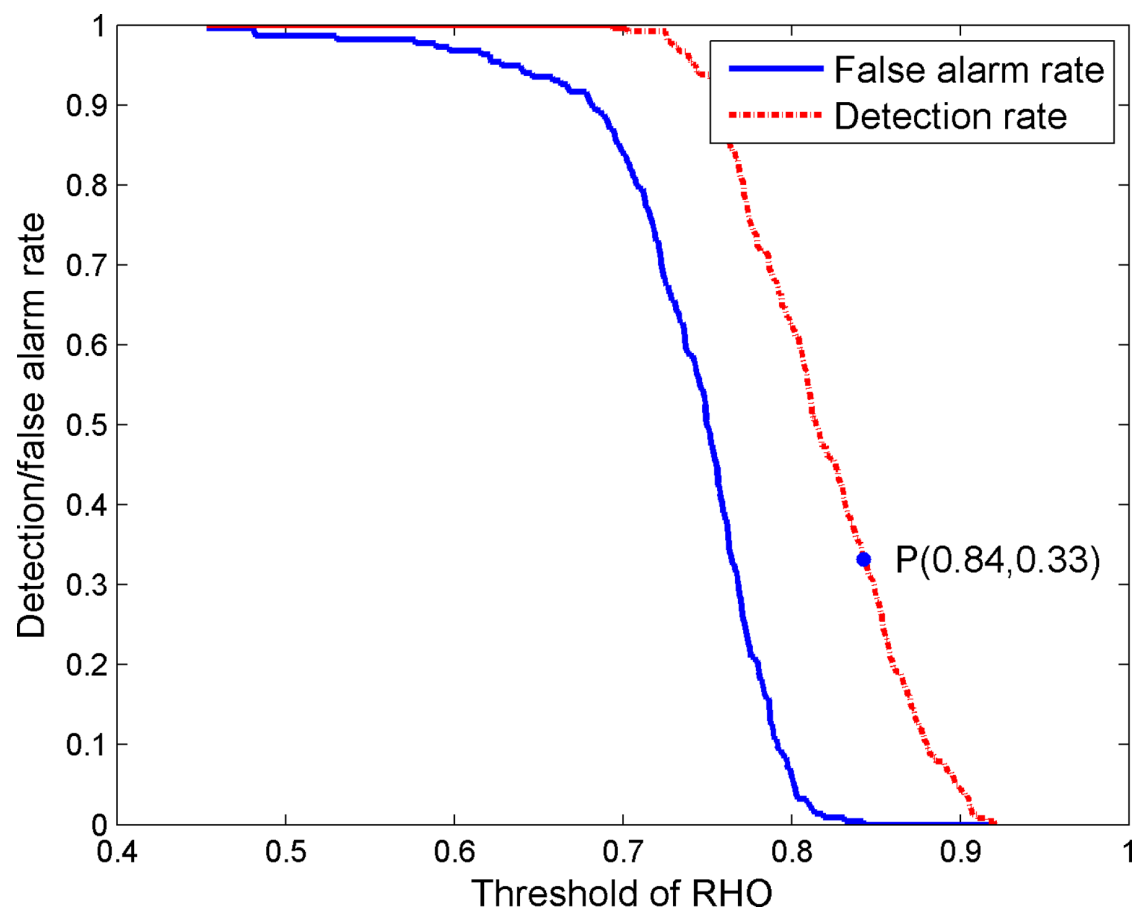

Figure 15c. Detection/false alarm rate of four methods. Point $P$ Is the highest detection rate when false alarm rate is zero.

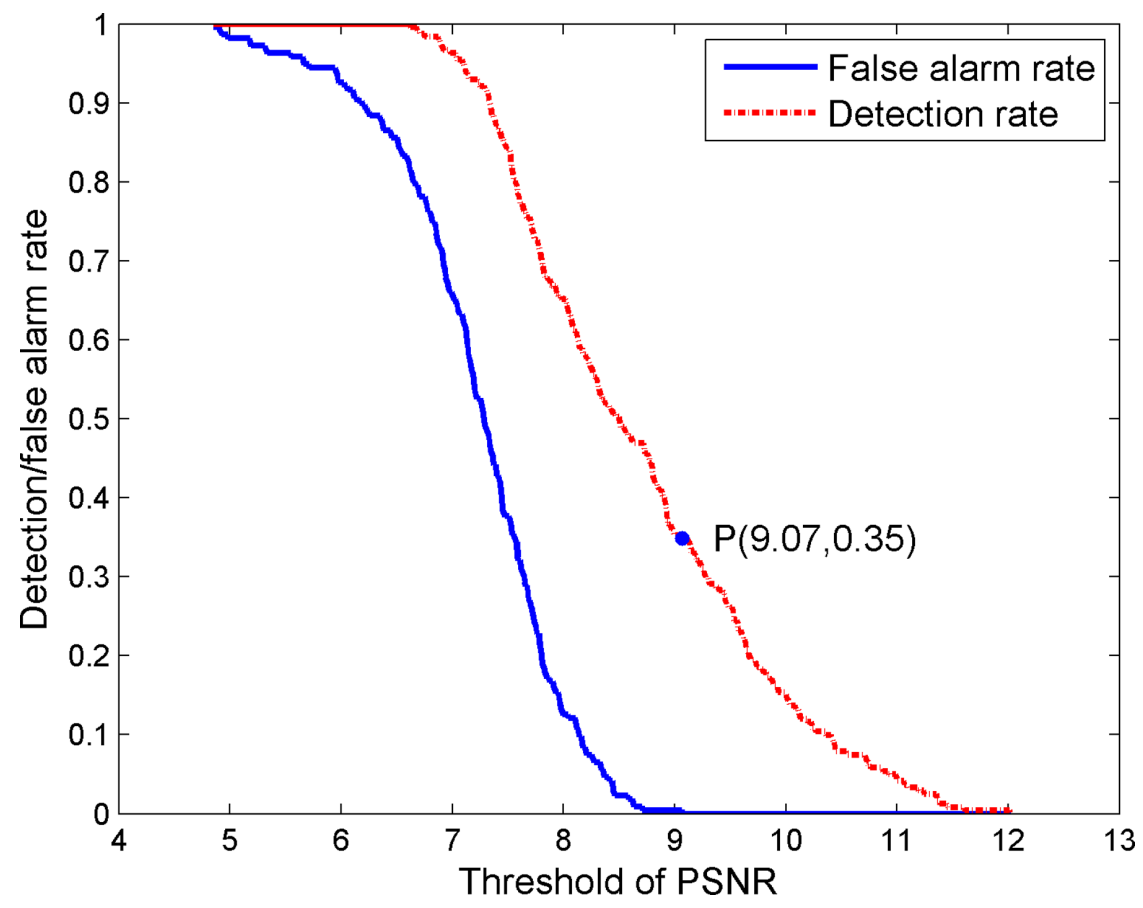


Figure 15d. Detection/false alarm rate of four methods. Point $P$ Is the highest detection rate when false alarm rate is zero.

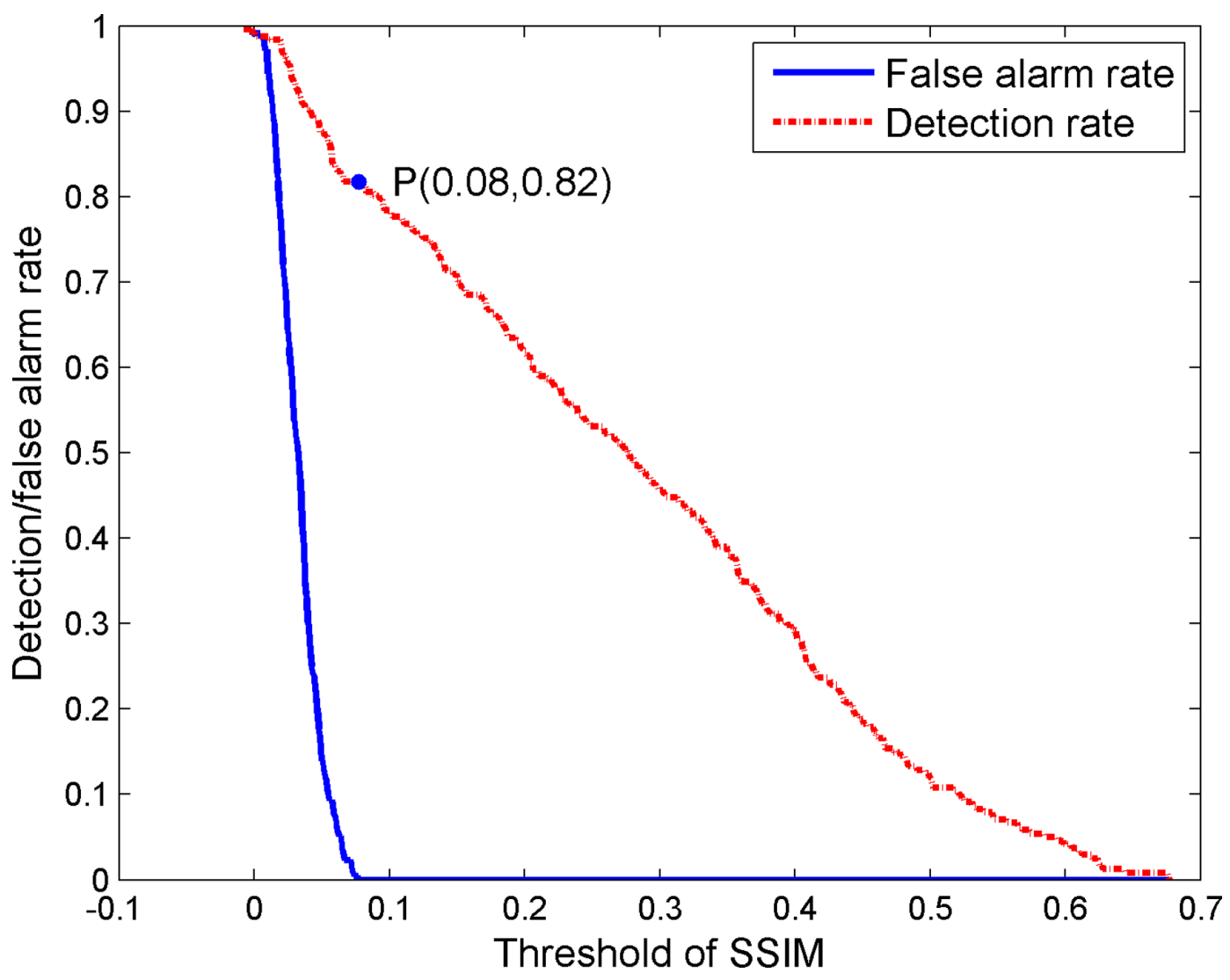




\section{CONCLUSION}

This paper presents a robust digital watermarking scheme for print and recapture process. Through periodic embedding of watermark, use self-convolution to synchronize, watermark information can be extracted accurately. The watermarking scheme presented in this paper is not limited to print and recapture process. Images containing watermark can be displayed on the display device, which is also possible to extract from the camera. There are two main contribution points, one is to propose a complete print-recapture watermark scheme which does not need the human interaction, and does not need the original image, the other is to give a practical application example of this scheme. There are still two aspects of the scheme to be improved. One is that the fidelity of the image needs to be improved. It can be seen from the experiment that PSNR is low. The other is that the computational complexity need to be reduced. A large amount of computation will reduce the practicality of the scheme due to the limited computing resources on the mobile phone.

\section{ACKNOWLEDGMENT}

This work was supported by the National Key Research and Development of China (No. 2016YFB0800404), the National Science Foundation of China (Nos. 61532005, 61332012), and the Fundamental Research Funds for the Central Universities (No. 2018JBZ001). 


\section{REFERENCES}

Anthony, T. S. Ho, \& Shu, F. (2003). A Print-and-scan Resilient Digital Watermark for Card Authentication. In Joint Conference of the 4th International Conference on Information, Communications and Signal Processing/4th Pacific-Rim Conference on Multimedia (pp. 1149-1152).

Cheng, D., Li, X., Qi, W., \& Yang, B. (2008). A Statistics-Based Watermarking Scheme Robust to Print-andScan. In International Symposium on Electronic Commerce and Security (pp. 894-898). IEEE Computer Society. doi:10.1109/ISECS.2008.68

Chiu, Y. C., \& Tsai, W. H. (2006). Copyright Protection against Print-and-Scan Operations by Watermarking for Color Images Using Coding and Synchronization of Peak Locations in Frequency Domain. Journal of Information Science and Engineering, 22(3), 483-496.

Kang, X., Huang, J., \& Zeng, W. (2010). Efficient general print-scanning resilient data hiding based on uniform log-polar mapping. IEEE Transactions on Information Forensics and Security, 5(1), 1-12. doi:10.1109/ TIFS.2009.2039604

Kutter, M. (1999). Watermarking resisting to translation, rotation, and scaling. Multimedia Systems and Applications, 3528, 423-432. doi:10.1117/12.337432

Lin, C. Y., \& Chang, S. F. (1999). Distortion modeling and invariant extraction for digital image print-and-scan process. In International Symposium on Multimedia Information Processing.

Pereira, S., \& Pun, T. (2000). Robust template matching for affine resistant image watermarks. IEEE Transactions on Image Processing, 9(6), 1123-1129. doi:10.1109/83.846253 PMID:18255481

Pramila, A., Keskinarkaus, A., \& Seppanen, T. (2008). Watermark robustness in the print-cam process. In Iasted International Conference on Signal Processing, Pattern Recognition and Applications (pp. 60-65). ACTA Press.

Solanki, K., Madhow, U., Manjunath, B. S., Chandrasekaran, S., \& El-Khalil, I. (2006). 'print and scan' resilient data hiding in images. IEEE Transactions on Information Forensics and Security, 1(4), 464-478. doi:10.1109/ TIFS.2006.885032

Wang, Z., Bovik, A. C., Sheikh, H. R., \& Simoncelli, E. P. (2004). Image quality assessment: From error visibility to structural similarity. IEEE Transactions on Image Processing, 13(4), 600-612. doi:10.1109/TIP.2003.819861 PMID:15376593

Yamada, T., \& Kamitani, M. (2013). A method for detecting watermarks in print using smart phone: finding no mark. In The Workshop on Mobile Video (pp. 49-54). ACM. doi:10.1145/2457413.2457425

Yu, L., Niu, X., \& Sun, S. (2005). Print-and-scan model and the watermarking countermeasure. Image and Vision Computing, 23(9), 807-814. doi:10.1016/j.imavis.2005.05.014

Zhang Mengmeng, female, master, studied signal and information processing at Beijing Jiaotong University in 2016. Her main research direction is digital watermarking and information hiding. Her graduation thesis is nondigital domain image watermarking technology. 